SECTION 2. Applied mathematics. Mathematical modeling.

Konstantin Anatolyevich Chuev Candidate of physical and mathematical Sciences, Assoc. prof., Gubkinsky branch of MSMU, Gubkin, Russia

kot111208@mail.ru

\title{
METHODS OF FINDING SOLUTIONS OF THE EQUATIONS OF HEAT AND MASS TRANSFER WITH THE USE OF SYNTHESIS OF INTEGRAL TRANSFORMATIONS
}

Abstract: To obtain approximate analytic solution of a system of linear equations of thermodynamics the technique with the use of synthesis of integral transformations (unilateral Laplace transform and the finite Fourier transform). The obtained approach to solution of the equations of the model are tested using the finite-difference technologies.

Key words: the heat and mass transfer, drying, potential migration, Laplace transform and the Fourier transform, convolution, integral equations, finite-difference.

\section{УДК 517.956}

\section{МЕТОДИКА НАХОЖДЕНИЯ РЕШЕНИЯ УРАВНЕНИЙ МОДЕЛИ ТЕПЛОМАССОПЕРЕНОСА С ИСПОЛЬЗОВАНИЕМ СИНТЕЗА ИНТЕГРАЛЬНЫХ ПРЕОБРАЗОВАНИЙ}

Аннотация: Для получения приближенно-аналитического решения системь уравнений линейной термодинамики предложена методика с использованием синтеза интегральных преобразований (одностороннего преобразования Лапласа и конечного преобразования Фурье). Адекватность полученного подхода для решения уравнений модели проверена с использованием конечно-разностных технологий.

Ключевые слова: тепломассоперенос, сушка, потеничиль переноса, преобразования Лапласа и Фурье, свертка, интегральные уравнения, конечноразностная схема.

Несмотря на широкое использование вычислительной техники для решения различных задач математической физики, интерес к алгоритмам и методам получения приближенных аналитических решений не ослабевает в силу возможности проведения анализа с физической точки зрения и получения инженерных методик.

Известно, что многие явления переноса могут быть до определенной степени точности описаны с помощью математического анализа линейной термодинамики [1, c. 24]. Например, сушка капиллярно-пористых тел является типичным нестационарным процессом, протекающим при наличии градиентов потенциалов переноса [2, с. 16]. Известные математические модели в теории сушки базируются в основном на диффузионно-фильтрационных представлениях о влагопереносе, которые формализируются в виде сопряженной системы линейных дифференциальных уравнений в частных производных А.В. Лыкова с соответствующими сопряженными граничными условиями, выражающие суперпозицию механизмов переноса потенциалов [3, с. 37]. За последние годы такие модели находят широкое применение для инженерных расчетов в сушильной технике, строительной теплофизике, в расчетах ряда химико-технологических процессов, а также разработке экспериментальных методов определения теплофизических свойств влажных материалов. 
Как правило, при математической формулировке явлений переноса в неподвижных капиллярно-пористых средах используют три искомых потенциала: локальные влагосодержание, температура и давление. Считаем, что геометрия тел обладает симметрией. Начальные условия задаются в виде постоянных значений потенциалов, и на оси симметрии отсутствует перенос субстанции. На границе тел рассматривается дифференциальный баланс теплоты как суперпозиция механизмов переноса теплопроводностью, конвективного теплообмена с окружающей средой с учетом фазовых превращений, а также дифференциальный баланс массы как суперпозиция механизмов переноса диффузией, термодиффузией, бародиффузией и конвективным массообменном. [4, с. 23]. В этом случае система принимает вид:

$$
\begin{gathered}
\frac{\partial U}{\partial F O}=k_{11} \nabla^{2} U+k_{12} \nabla^{2} T+k_{13} \nabla^{2} P ; \\
\frac{\partial T}{\partial F O}=k_{21} \nabla^{2} U+k_{22} \nabla^{2} T+k_{23} \nabla^{2} P ; \\
\frac{\partial P}{\partial F O}=k_{31} \nabla^{2} U+k_{32} \nabla^{2} T+k_{33} \nabla^{2} P ; \\
U(X, 0)=T(X, 0)=P(X, 0)=0 ; \\
\frac{\partial U(0, F o)}{\partial X}=\frac{\partial T(0, F o)}{\partial X}=\frac{\partial P(0, F o)}{\partial X}=0 ; \\
\frac{\partial U(1, F o)}{\partial X}+B i_{m} \cdot[U(1, F O)-1]=\frac{\partial T(1, F o)}{\partial X}+B i_{q} \cdot[T(1, F O)-1]= \\
=\frac{\partial P(1, F o)}{\partial X}+B i_{P}[P(1, F o)-1]=0 ; \\
\nabla^{2}=\frac{\partial^{2}}{\partial X^{2}} ;
\end{gathered}
$$

где $F o-$ безразмерный критерий Фурье- $F o \in(0, \infty) ;, k_{\text {iу }}$-постоянные коэффициенты переноса, $B i_{m}, B i_{q}, B i p$ - массообменное, теплообменное и фильтрационные числа Био. Будем идентифицировать безразмерные U,T,P-относительные влагосодержание, температура и давление для неограниченной пластины ( $X \in[0,1]$.).

Последовательным применением одностороннего преобразования Лапласа [5, с. 31] по переменной $F o$ и конечного косинус-преобразования Фурье по переменной $X$ с характеристическим уравнением $\lambda_{k} \cdot \operatorname{tg} \lambda_{k}=1 / B i_{q}$ система (1)-(6) становится алгебраической относительно изображений потенциалов, после решения которой получен результат в векторной форме в пространстве оригиналов с неизвестными $U(1, F o), P(1, F o)$ :

$$
\begin{aligned}
& \bar{\Phi}(X, F o)=1+2 \sum_{k=1}^{\infty} \frac{\left(\lambda_{k}^{2}+B i_{q}^{-2}\right) \cdot \cos \left(\lambda_{k} X\right)}{\left(\lambda_{k}^{2}+B i_{q}^{-1}+B i_{q}^{-2}\right)} \cdot\left\{\left[\left(B i_{q}^{-1}-B i_{m}^{-1}\right) \cdot \int_{0}^{F o} \varphi_{1}(F o-Y) \cdot U(1, Y) d Y\right] \times\right. \\
& \left.\quad \times \cos \lambda_{k}+\frac{\sin \lambda_{k}}{\lambda_{k}} \cdot \overline{\varphi_{2}}(F o)+\left[\left(B i_{q}^{-1}-B i_{P}^{-1}\right) \cdot \int_{0}^{F o} \overline{\varphi_{3}}(F o-Y) \cdot P(1, Y) d Y\right] \cdot \cos \lambda_{k}\right\} .
\end{aligned}
$$

где

$$
\begin{aligned}
& \bar{\Phi}(X, F O)=[U(X, F O), T(X, F O), P(X, F O)]^{T} ; \\
& \overline{\varphi_{i}}(F O-Y)=\left[\varphi_{1 i}(F O-Y), \varphi_{2 i}(F O-Y), \varphi_{3 i}(F O-Y)\right]^{T}, \\
& \overline{\varphi_{2}}(F O)=\left[\varphi_{12}(F O), \varphi_{22}(F O), \varphi_{32}(F O)\right]^{T} ; \\
& \varphi_{i j}(F O-Y)=A_{i j} \exp \left[k_{1}(F O-Y)\right]+B_{i j} \exp \left[k_{2}(F O-Y)\right]+C_{i j} \exp \left[k_{3}(F O-Y)\right] ; \quad i=1,3 \\
& k_{1}, k_{2}, k_{3} \text {-корни уравнения: } \\
& s^{3}+a \lambda_{k}^{2} s^{2}+b \lambda_{k}^{4} s+c \lambda_{k}^{6}=0
\end{aligned}
$$


$A_{i j}, B_{i j}, C_{i j}, a, b, c$-постоянные коэффициенты, зависящие от определяющих критериев задачи.

Если в (7) положить $X=1$, то получим систему интегральных уравнений типа Вольтера[6, с. 42], относительно $U(1, F o), T(1, F o), P(1, F o)$. Вновь переходя к изображениям по Лапласу, решение при переходе к оригиналам, с учетом свертки [5], имеет вид:

$$
\bar{\Phi}(1, F O)=L^{-1}\left[\frac{\overline{P_{5}}\left(\lambda_{k}, s\right)}{Q_{6}\left(\lambda_{k}, s\right)}\right]=\sum_{i=1}^{6} \overline{A_{i j}}\left(\lambda_{k}\right) \cdot \exp \left(l_{i} F O\right) ; \quad j=1,3
$$

где $\quad \overline{P_{5}}\left(\lambda_{k}, s\right)=\left[P_{5_{1}}\left(\lambda_{k}, s\right), P_{5_{2}}\left(\lambda_{k}, s\right), P_{5_{3}}\left(\lambda_{k}, s\right)\right]^{T} ; \quad P_{5 j}\left(\lambda_{k} ; s\right), Q_{6}\left(\lambda_{k} ; s\right)-\quad$ соответственно полиномы пятой и шестой степени $s$ с коэффициентами, зависящими от $\lambda_{k} ; l_{i}$-корни уравнения $Q_{6}\left(\lambda_{k}, s\right)=0 ; \overline{A_{i j}}\left(\lambda_{k}\right)=\left[A_{i 1}\left(\lambda_{k}\right), A_{i 2}\left(\lambda_{k}\right), A_{i 3}\left(\lambda_{k}\right)\right]^{T}$ - вектор постоянных. С учетом (9) выражение (7) является решением системы (1)-(6), причем верхний предел суммы, заменяется на конечное число $N$ для обеспечения заданной точности. Исходя из физического смысла задачи, корни уравнения (8) должны быть либо тремя действительными отрицательными, либо одним отрицательным и двумя комплексносопряженными с отрицательной действительной частью. Аналогичные рассуждения применимы и для корней $l_{i}$ уравнения $Q_{6}\left(\lambda_{k}, s\right)=0$.

Используя для безразмерных критериев, диапазоны изменения, полученные на основе анализа физико-химических свойств ряда капиллярно-пористых материалов [7, c. 53] показано, что корни характеристических уравнений в подавляющем своем большинстве удовлетворяют наложенным условиям физичности. Это означает, что система (1)-(3) может быть классифицирована как система уравнений в частных производных параболического типа по Хеллвигу [8, с. 18].

Теперь систему (1)-(6) интегрировали численно по маршевой относительно Fo конечно-разностной схеме [9, с. 21]. На дискретной области с узлами $F_{O_{i}}=i \cdot \Delta F o, X_{j}=j \cdot \Delta X$, где $\Delta X=1 / m$. Для аппроксимации уравнений модели выбран четырехточечный шаблон, дискретный аналог уравнений модели с первым порядком точности по $\Delta F O$ и вторым по $\Delta X$. Причем для сходимости и устойчивости вычислительного процесса соотношение между шагами интегрирования по безразмерному времени и координате выбиралось по условию $\Delta F o<<\Delta X$ [10, с. 53]. Для тех же исходных данных, были получены результаты по кинетике процесса, представленные на рис.1.

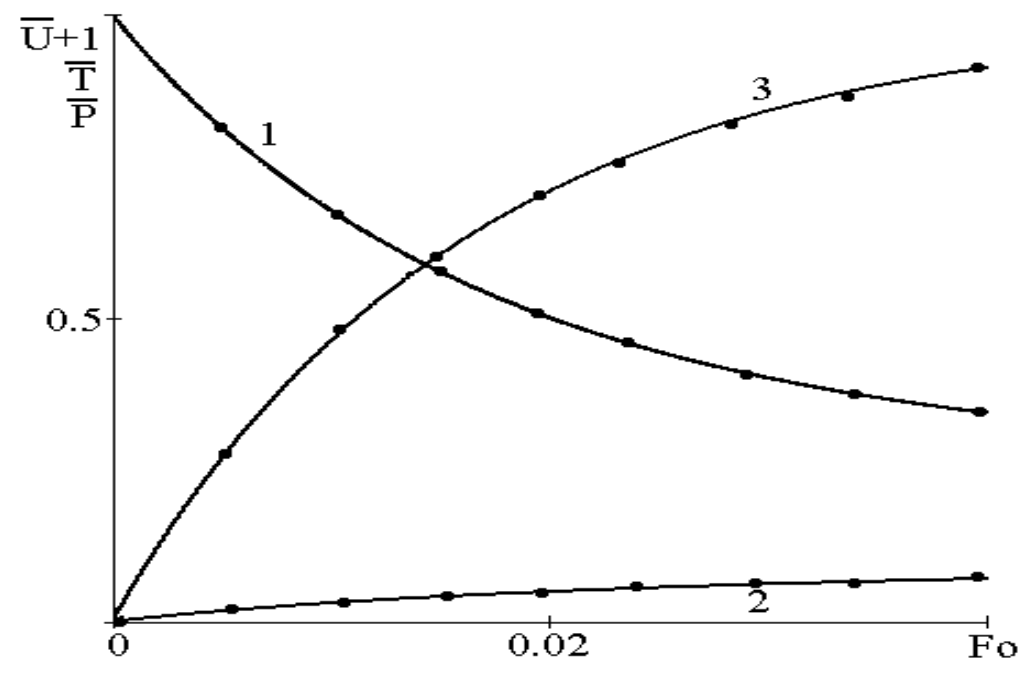

Рисунок 1 - Результаты вычислительного эксперимента: $1-U+1 ; 2-T ; 3-P$ 
Аналитическое решение уравнений модели представлены формулой (7) в виде сходящихся рядов, удобно для практических расчетов. В суммах можно ограничится до 8 членов ряда, из-за их быстрой сходимости. Численное решение уравнений модели адекватно согласуются спецификой процессов сушки в капиллярно-пористых телах, что интерпретируется на рис.1. и полученным аналитическим решением (на рис.1непрерывные кривые). Предложенный подход может быть вполне адаптирован для других геометрий. Подход позволяет избежать использование не вполне обоснованных численных технологий в смысле аппроксимации, устойчивости и сходимости.

\section{References:}

1. Лыков А. В. Тепломассообмен: (Справочник) - М.: Энергия, 1978. - 480 с.

2. Лыков А.В., Михайлов Ю.А. теория тепло- и массопереноса.- М.-Л.: Госэнергоиздат, 1963.- 535 c.

3. Карташов Э.М. Аналитические методы в теории теплопроводности твердых тел. М.: Высшая школа, 2001. - 550 с.

4. Беляев Н.М., Рядно А.А. Методы теории теплопроводности. Ч.1.-М.: Высшая школа, 1982.- 327 с.

5. Деч Г. Руководство к практическому применению преобразования Лапласа и zпреобразования. - М.: Физматгиз, 1971. 288 с.

6. Петровский Н.Г. Лекции по интегральным уравнениям .- М.: Наука, 1965. - 246 с.

7. Лыков А.В. Теория сушки.- М.: Энергия, 1968. - 472 с.

8. Фарлоу С. Уравнения с частными производными для научных работников и инженеров.- М.: Мир, 1985.- 384 с.

9. Самарский А.А., Николаев Е.С. Методы решения сеточных уравнений. - М.: Наука, 1978. - 592 c.

10. Самарский А.А., Вабищевич П.Н. Численные методы решения задач конвекциидиффузии.- М.: Наука, 1999.- 247 с. 